\title{
Cardiovascular Disorders during HIV-AIDS in the Point G University, Hospital Internal Medical Service
}

\author{
Camara BD1, Traore AK1, Soukho-Kaya A1, Traore D1, Dembele M11, Diall IB2,

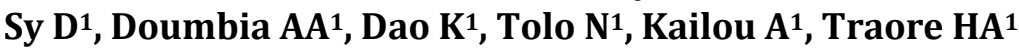 \\ ${ }^{1}$ Department of Internal Medicine CHU Point G, Bamako, Mali \\ ${ }^{2}$ Cardiology Department CHU of Point G, Bamako, Mali \\ Email: bouadaoudcamara@yahoo.fr, traoreak@gmail.com
}

How to cite this paper: Camara $\mathrm{BD}$, Traore AK, Soukho-Kaya A, Traore Djennebou, Dembele M, Diall IB, Sy D, Doumbia AA, Dao K, Tolo N, Kailou A and Traore HA (2020) Cardiovascular Disorders during HIV-AIDS in the Point G University, Hospital Internal Medical Service. Open Journal of Internal Medicine, 10, 13-20.

https://doi.org/10.4236/ojim.2020.101002

Received: November 19, 2019

Accepted: January 12, 2020

Published: January 15, 2020

Copyright $\odot 2020$ by author(s) and Scientific Research Publishing Inc. This work is licensed under the Creative Commons Attribution International License (CC BY 4.0).

http://creativecommons.org/licenses/by/4.0/

(c) (i) Open Access

\begin{abstract}
The number of HIV-infected patients in the world is estimated at 34 million, including 30.1 million adults with 2.7 million new infections and 1.8 million deaths from HIV according to the 2011 UN AIDS report [1]. The prevalence of HIV infection in the general population of Mali is estimated at $1.1 \%$ according to DHSM-V [2]. Cardiovascular manifestations are increasingly encountered during our consultations with patients living with HIV. Thus we initiated this work with the aim of studying the cardiovascular lesions during the HIV-AIDS in the department of internal medicine of the point G. Methods: This retrospective descriptive study was conducted in the Internal Medicine department of the University Hospital Center (CHU) of the Point G from January 2008 to December 2012. Having included all HIV + patients hospitalized in the service with cardiovascular manifestations and who had performed a chest X-ray, cardiac and vascular ultrasound, electrocardiogram and/or cerebral computed tomography. Results: At the end of this work, 20 patients with cardiovascular lesions were identified in 273 hospitalized patients during the study period, i.e. a frequency of $7.3 \%$. The 40 to 50 age group accounted for $45.0 \%$ with an average age of $42.4 \pm 8.73$ years. The most common cardiovascular risk factors were hypertension and active smoking with $31.58 \%$ each. Pericarditis accounted for $32 \%$ of cases, followed by cardiomyopathies with $23 \%$. Conclusion: In our study, cardiovascular lesions are common in PHAs and etiologies are dominated by pericarditis.
\end{abstract}

\section{Keywords}

Cardiovascular Disorders, HIV-AIDS, ARVs 


\section{Introduction}

HIV/AIDS infection is a global pandemic that affects almost all countries.

The number of HIV-infected patients in the world is estimated at 34 million, including 30.1 million adults; 2.7 million new infections and 1.8 million deaths from HIV according to the 2011 UN AIDS report [1].

Mali has been confronted with the problem of HIV infection since the first case of AIDS was reported in 1985.

Since 2001, the response to the epidemic has taken a multi-sectoral dimension and is coordinated by the National High Council for the Fight against AIDS, chaired by the President of the Republic. The prevalence of HIV infection in the general population of Mali is estimated at $1.1 \%$ by DHS V.

The seroprevalence rate among women aged $15-49$, estimated at $1.3 \%$, is slightly higher than that observed for men in the same age group $(0.8 \%)$. In addition, the prevalence of HIV is lower in rural areas $(0.9 \%)$ than in urban areas (2.0\%) and in Bamako (1.7\%) [2].

HIV infection is not only responsible for immunodeficiency leading to opportunistic infectious diseases and neoplasia (non-Hodgkin's lymphoma, Kaposi's disease, cerebral lymphoma, cervical cancer). It is also accompanied by organ damage attributable to the virus itself and certain metabolic functions.

These biological and/or clinical abnormalities may be associated with those induced by powerful antiretroviral multi-therapy [3].

Since 1996, Highly Active Antiretroviral Therapy (HAART) has been available in industrialized countries, making this serious infection a chronic disease in these countries.

This antiretroviral therapy usually involves 3 drugs: 2 nucleoside analogues of reverse transcriptase and an anti-protease or non-analogue reverse transcriptase leading to a dramatic reduction in morbidity and mortality related to HIV infection.

Concomitant with the use of this antiretroviral treatment appeared metabolic disorders (dyslipidemia, insulin resistance, lipodystrophy) that can lead to the occurrence of cardiovascular complications such as: myocardial infarction, arteriopathy of the lower limbs and stroke HTA, myocarditis, pericarditis in a population under 50 years of age.

Thus these complications became the 3 - 4th cause of death and hospitalization of HIV-infected patients in industrialized countries after AIDS and infectious, oncologic and hepatic causes [4].

The risk of arterial thrombosis or venous thrombosis is very high in HIV-infected patients.

Thus, an increased incidence of venous thromboembolic events under anti proteases has been reported by some authors [5].

$\mathrm{HIV}$-infected patients have a higher risk of coronary disease than the general population with a 10 -year Framingham risk score greater than $20 \%$, twice as high as in the uninfected population [6].

It appears that in $\mathrm{HIV}$-infected patients the first episode of acute coronary 
syndrome can occur earlier, about ten years earlier than in non-HIV-infected patients [7].

\section{Methodology}

This was a retrospective descriptive study conducted from January 2008 to December 2012 in the Department of Internal Medicine at the University Hospital Center (CHU) of Point G.

In our study, we defined criteria for inclusion and non-inclusion of patients.

\subsection{Inclusion Criteria}

All HIV patients with at least one cardiovascular disease.

Patients with HIV who performed all complementary confirmatory examinations of cardiovascular impairment (chest X-ray, cardiac and vascular ultrasound, ECG cerebral CT.

\subsection{Criteria of Non-Inclusion}

Patients with HIV without cardiovascular involvement.

Patients with HIV who could not do a chest X-ray, echocardiogram, electrocardiogram, lower extremity Doppler and/or cerebral CT.

For all patients, we collected variables.

Clinics: socio-demographic (age, sex, ethnicity, profession, residence).

The search for functional signs: cough, dyspnea, unilateral pain of the lower limb.

General condition, skin and lower limb aspects, weight and height with calculation of BMI.

Physical signs: IMO, neurological deficits.

Biological:

NFS, CRP, fasting glucose, HIV serology, total cholesterol, triglycerides, LDL-c, HDL-c, transaminases, serum uric acid, troponins, D-dimer, serum creatinine, CD4 LT level, viral load.

Morphology:

X-ray of the thorax, ECG, Doppler echo of cardiac and vessels, cerebral CT.

These data were collected on an individual survey form.

Data entry and analysis was done on Word 2007 and Epi Info 3.5.3 software.

\section{Results}

\subsection{Overall Results}

From January 2008 to December 2012 we included 20 patients out of $273 \mathrm{im}$ munocompromised patients with HIV, a global prevalence of cardio-vascular disorders estimated at $7.3 \%$.

\subsection{Sociodemographic, Clinical and Para Clinical Data}

The average age of our patients was $42.4 \pm 8.73$ years with extreme ages of 20 
and 60 years.

Age range between 41 to 50 was more represented with $45 \%$ or 9 cases/20.

The average duration of hospitalization was 42.75 days (Figure 1 ).

The sex ratio was 0.81 or 9 men out of 11 women.

HTA and active smoking accounted for $31.58 \%$, respectively, for our patients (Table 1).

Pericarditis accounted for $32 \%$ of cases, followed by cardiomyopathies with $23 \%$ of cases (Table 1 ).

Women accounted for $55.0 \%$ of cases with a ratio of 0.81 (Table 1 ).

Tachycardia was observed in $65.0 \%$ of patients (Table 1 ).

Most of our patients were at stage C3 (CDC/WHO), which is $80.0 \%$ of cases (Table 1).

The CD4 LT level below $200 \mathrm{cel} / \mathrm{mm}^{3}$ in $75.0 \%$ of cases (Table 1).

$90 \%$ of patients had anemia (Table 1 ).

The systolic ejection fraction of LV was impaired in $42.86 \%$ of patients (Table 1).

Nearly half of the patients died during the course of the evolution (Table 1).

\section{Discussion}

Cardiovascular damage in general is often observed in HIV patients and in particular heart disease which affects all tunics of the heart.

Our retrospective study presented limitations including the lack of accessibility of all HIV patients to all complementary examinations for the diagnosis of cardiovascular disease and this especially as all these exams are not part of the kit. free of HIV patients.

In our study, the prevalence of cardiovascular disease was 7.3\%.

This rate is higher than that reported by AULAGNIER 5.8\% [8] and that of the Burkinabe study 2.7\% [9].

The CD4 count was below $200 \mathrm{cel} / \mathrm{mm}^{3}$ in $75 \%$.

Severe anemia was found in $40 \%$ of patients.

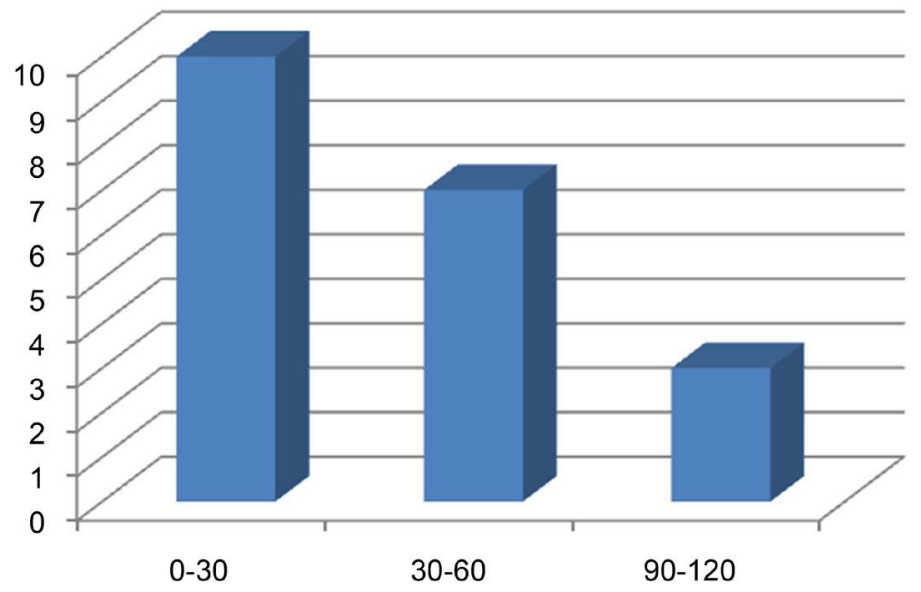

Série1

Figure 1. Distribution of patients by length of hospital stay. 
Table 1. distribution of patients by cardiovascular risk factor, clinical, and paraclinical.

\begin{tabular}{|c|c|c|}
\hline Variable & Numbers & $(\%)$ \\
\hline \multicolumn{3}{|l|}{ Sex } \\
\hline Female & 11 & 55.00 \\
\hline \multicolumn{3}{|l|}{ Cardiovascular risk factors } \\
\hline Active smoking & 6 & 31.58 \\
\hline HTA & 6 & 31.58 \\
\hline $\begin{array}{c}\text { Clinical manifestations } \\
\text { Alteration of the general condition } \\
\text { Cardiac frequency }\end{array}$ & 5 & 25 \\
\hline Tachycardia & 13 & 65.00 \\
\hline \multicolumn{3}{|l|}{ HIV serology } \\
\hline$H I V 1$ & 16 & 80 \\
\hline \multicolumn{3}{|l|}{ Cardiovascular discorders } \\
\hline \multicolumn{3}{|l|}{ Ejection fraction (\%) } \\
\hline$<45 \%$ & $6(/ 14)$ & 42.86 \\
\hline \multicolumn{3}{|l|}{$H b(g / d)$} \\
\hline Anemia & 18 & 90.00 \\
\hline \multicolumn{3}{|l|}{ CDClWHO Classification } \\
\hline $\mathrm{C} 3$ & 16 & 80.00 \\
\hline \multicolumn{3}{|l|}{ CD4 LT level } \\
\hline$<200$ & 15 & 75.00 \\
\hline \multicolumn{3}{|l|}{ Evolution } \\
\hline Death & 9 & 45.00 \\
\hline
\end{tabular}

This rate is significantly higher than that reported by the Burkinabe study $31 \%$ [9].

The deterioration of the general condition was present in $25 \%$ of cases.

This prevalence is similar to that reported by the Ivorian study $28.2 \%$ [10].

The average age of our patients was 42.4 years \pm 8.73 with extremes of 20 and 60 years, this rate is presumable to that reported by the Ivorian study $37.9 \pm 13$ [10].

The dyspnea accounted for $10 \%$ of cases, this rate is significantly lower than that reported by the Ivorian study $67.6 \%$ [10].

The mean hospital stay in our study was 42.75 days.

This duration is greater than that reported by the Chadian study which was 30.5 days $[11]$.

During the hospitalization, we recorded 9 deaths (45\%) including 6 men and 3 women this rate is higher than that found by the Chadian study $12.7 \%$ [11].

Tachycardia was found in $65 \%$ of patients, which is similar to that reported by DIARRA 67.43\% [12].

Cardiomegaly was found in $37.5 \%$ of patients. 
This prevalence is higher than that reported by DIARRA 28.78\% [12].

In our study, pericardial involvement accounted for $32 \%$ of cases.

This prevalence is within the range of Western studies estimated between 10 and $59 \%$ with an average of $20 \%$ [13].

It is superimposable to that reported by the Congo Brazzaville study with $35.3 \%$ of cases [14].

In our study, we found a predominance of HIV 1 in $80 \%$ of cases.

This rate is similar to that reported by SIDIBE $82 \%$ [15].

In our study, cardiomyopathies accounted for $23 \%$ of cases.

This prevalence is similar to that reported by the Cameroonian study with $23.3 \%$ of cases [16].

The prevalence of stroke in our study was $9 \%$ of cases.

This prevalence is significantly lower than that reported by OVBIAGELE et al. with $67 \%$ of cases [17].

In our study, the frequency of venous thrombosis and AOMI was $9 \%$ and $4 \%$ each.

This prevalence is identical to that found by LIJFERING et al. with $10 \%$ and $6 \%$ each [18].

The most common cardiovascular risk factors associated with HIV were active smoking and hypertension with $31.58 \%$ each.

This prevalence of active smoking is comparable to that reported by the sub-Saharan Africa study with $29.7 \%$ of cases for Mali [19].

\section{Conclusions}

HIV infection and antiretroviral therapy are responsible for cardiovascular complications.

These are capable of aggravating an already heavy prognosis.

In our study, cardiovascular lesions are common in PHAs and etiologies are dominated by pericarditis.

Cardio-vascular damage is relatively common.

Our study showed that pericarditis accounted for $32 \%$ of cases.

Risk factors were active smoking and HTA 30\% each.

The ARV protocol using D4T or AZT + 3TC + NVP or EFV was used in most patients $35 \%$.

The correct management of cardiovascular risk factors, the early detection of metabolic abnormalities and their rapid correction, the early initiation of ARVs and the regular monitoring of the HIV patient, the choice of less toxic molecules on the cardiovascular system are in addition measures to reduce cardiovascular damage and improve the prognosis in the short and medium term.

The availability of complementary tests and their easy and free access to patients are essential to prevent these HIV-related complications.

\section{Conflicts of Interest}

The authors declare no conflicts of interest regarding the publication of this paper. 


\section{References}

[1] World Day Aids (2011) Epidemiological Data. UNAIDS Report, 12 p.

[2] (2013) Demographic and Health Survey of Mali (DHSM-V). Preliminary Report on HIV Prevalence. INFO-STAT, Planning and Statistics Unit (CPS), National Institute of Statistics (INSTAT), Bamako, and ICF International, Calverton, $16 \mathrm{p}$.

[3] Hoen, B. (2007) Role of HIV in Bone, Cardiovascular and Renal Complications. In: Hoen, B., Martin, I.P. and Boccara, F., Eds., Medicine and Infectious Diseases, Vol. 37, Elsevier, France, 1-12.

[4] Kwong, G.P.S., Azra, C.G., Rode, R.A., Bartley, L.M., Cowling, B.J., Da Silva, B., et al. (2006) Comparison of the Risks of Atherosclerotic Events versus Death from Others Causes Associated with Antiretroviral Use. AIDS, 20, 1942-1950. https://doi.org/10.1097/01.aids.0000247115.81832.a1

[5] Laurence, J. (1998) Vascular Complications Associated with the Use of HIV Protease Inhibitors. The Lancet, 351, 1960. https://doi.org/10.1016/S0140-6736(05)78645-1

[6] Bergersen, B.M., Sandvik, L., Bruun, J.N. and Tonstad, S. (2004) Elevated Framingham Risk Score in HIV-Positive Patients on Highly Active Antiretroviral Therapy: Results from a Norwegian Study of 721 Subjects. European Journal of Clinical Microbiology \& Infectious Diseases, 23, 625-630. https://doi.org/10.1007/s10096-004-1177-6

[7] Knudsen, A. (2013) Angiographic Features and Cardiovascular Risk Factors in Human Immunodeficiency Virus-Infected Patients with First-Time Acute Coronary Syndrome. American Journal of Cardiology, 111, 63-67. https://doi.org/10.1016/j.amjcard.2012.08.047

[8] Jerome, A. (2007) Cardiovascular Complications of the HIV-Infected Subject. Synthesis of the Literature Descriptive Study within a Population Followed at the University Hospital of Saint-Germain-en-Lay. Med. These, $92 \mathrm{p}$.

[9] Niakara, A., Drabo, Y.J., Kambire, Y., Nebie, L.V.A., Kabore, N.J.P. and Simon, F. (2002) Cardiovascular Disease and HIV Infection: A Study of 79 Cases at the CHN of Ouagadougou (Burkina Faso). Bulletin de la Société de Pathologie Exotique, 95, 23-26.

[10] Anzouan-Kacou, J.B., Dogoua, P., Konin, C., Coulibaly, I., Ouattara, I., Eholie, S.P., et al. (2012) Cardiovascular Disorders in HIV-Positive Patients Not Treated with Antiretrovirals. Tropical Cardiology, 131, 22 p.

[11] Mouanodji, M. (1996) Clinical Profile of 55 AIDS Patients with Cardiac Manifestations in Africa: Experiments at the N'djamena Central Hospital (Chad). African's Black Medicine, 43, 273-278.

[12] Diarra, A. (2000) Prevalence of Cardiovascular Diseases during AIDS in the Cardiology Departments of Gabriel Touré and G-Spot Hospitals; about 132 Cases. Med. These, Bko, $40 \mathrm{p}$.

[13] Bennis, A., Mehadji, B., Nourredine, M., et al. (1995) Cardiac Tamponade in the Acquired Immunodeficiency Syndrome. Tropical Cardiology, 21, 87-90.

[14] Bouramoue, C. and Ekoba, J. (1996) Heart and AIDS. Tropical Medicine, 56, 33-39.

[15] Sidibé, S. (2007) Pericarditis during HIV Infection in Bamako. These, Med, Bko, N64.

[16] Nzuobontane, D., Blackett, K.N. and Kuaban, C. (2002) Cardiac Involvement in HIV Infected People in Yaounde, Cameroon. Postgraduate Medical Journal, 78, 678-681. https://doi.org/10.1136/pmj.78.925.678 
[17] Ovbiagele, B. (2011) Increasing Incidence of Ischemic Stroke in Patients with HIV Infection. Neurology, 76, 444-450. https://doi.org/10.1212/WNL.0b013e31820a0cfc

[18] Lijfering, W.M., Sprenger, H.G., Georg, R.R., Van Der Meulen, P.A. and Van Der Meer, J. (2008) Relationship between AIDS and Thrombophilic Abnormalities in HIV Infection. Clinical Chemistry, 54, 1226-1233.

https://doi.org/10.1373/clinchem.2008.103614

[19] Jaquet, A., Bissagnene, E., Ekouevi, D.K., Traore, A., abubakrine, M., Maiga, M., et al. (2009) Tobacco Use and Its Determinants in HIV-Infected Patients on Antiretroviral Therapy in West African Countries. International Journal of Tuberculosis and Lung Disease, 13, 1433-1439. 